\title{
Dihydrolipoamide dehydrogenase suppression induce human tau phosphorylation by increasing whole body glucose levels in a $C$. elegans model of Alzheimer's Disease.
}

\begin{abstract}
The microtubule associated tau protein becomes hyperphosphorylated in Alzheimer's disease (AD). While hyperphosphorylation promotes neurodegeneration, the cause and consequences of this abnormal modification are poorly understood. As impaired energy metabolism is an important hallmark of $\mathrm{AD}$ progression, we tested whether it could trigger phosphorylation of human tau protein in a transgenic $C$. elegans model of AD. We found that inhibition of a mitochondrial enzyme of energy metabolism, dihydrolipoamide dehydrogenase (DLD) resulted in elevated whole-body glucose levels as well as increased phosphorylation of tau. Hyperglycemia and tau phosphorylation were induced by either epigenetic suppression of the dld-1 gene or by inhibition of the DLD enzyme by the inhibitor, 2-methoxyindole-2carboxylic acid (MICA). Although the calcium ionophore A23187 could reduce tau phosphorylation induced by either chemical or genetic suppression of DLD, it was unable to reduce tau phosphorylation induced by hyperglycemia. While inhibition of the $d l d-1$ gene or treatment with MICA partially reversed the inhibition of acetylcholine neurotransmission by tau, neither treatment affected tau inhibited mobility. Conclusively, any abnormalities in energy metabolism were found to significantly affect the AD disease pathology.
\end{abstract}




\section{Introduction:}

Mitochondria plays an important role in cell bioenergetics and survival while mitochondrial dysfunction proposed as a key mediator of neurodegenerative diseases such as Alzheimer's disease (AD) (1-3). In clinical studies, decline in cerebral glucose utilization, glucosedependent energy production and associated enzyme's activities such as pyruvate dehydrogenase $(\mathrm{PDH})$ and $\alpha$-ketoglutare dehydrogenase $(\mathrm{KGDH})$ are observed in $\mathrm{AD}$ patients, which highlights the importance of glucose energy metabolism in disease progression (4-8).

Abnormal glucose levels could be linked to formation of neurofibrillary tangles (NFTs), aggregates of hyper-phosphorylated tau protein that is diagnostic features of advanced $\operatorname{AD}(9,10)$. Tau regulates, maintains and stabilizes microtubule assembly under normal physiological conditions. Under such conditions, an average of 2-3 phosphate groups is present on each tau molecule, whereas in the $\mathrm{AD}$ brain the level of tau phosphorylation is 3-4 times higher $(11,12)$. Hyperphosphorylation leads to self-aggregation of tau filaments and activation of caspase-3 in vitro. In vivo, hyperphosphorylation is associated with tissue deterioration and cell death $(13,14)$.

Tau phosphorylation is negatively regulated by another post-translational modification $O$-GlcNAcylation of serine or threonine residues by UDP-GlcNAc at sites that overlap phosphorylation sites $(15,16)$. Given that UDP-GlcNAc biosynthesis is impaired by glucose catabolism, glucose catabolism could also cause reduced $O$-GlcNAcylation. As a result, down regulation of O-GlcNAc by low glucose levels is expected to promote tau hyperphosphorylation $(17,18)$. Although it has not yet been determined whether low glucose levels are a cause or consequence of $\mathrm{AD}$, the relationship between glucose levels, $O$ GlcNAcylation and tau hyperphosphorylation provides a plausible causal mechanism. Indeed, a drop in glucose energy metabolism has been proposed to minimize AD-associated pathology, but the authors of the study proposed a mechanism in which suppression of energy metabolism compensates for the reduced nutrient and oxygen supply in the neuronal microenvironment (19). Moreover, improved prognosis against AD symptoms observed in several model systems under caloric restriction or reducing glucose-dependent energy production also supports the hypothesis that decrease in glucose metabolism may be a protective measure against $\mathrm{AD}(20-22)$. 
Dihydrolipoamide dehydrogenase $(d l d)$ encodes a core metabolic enzyme and a part of two major enzyme complexes that contribute directly to glucose energy metabolism, PDH and KGDH (23). Remarkably, four single nucleotide variants of the $d l d$ gene are associated with increased risk of late-onset $\mathrm{AD}$, though how each variant specifically affects DLD activity is still unknown (24). Interestingly, in brains from Swedish AD patients with the APP670/671 mutation $A \beta$ was overproduced and all subunits of KGDH were suppressed except DLD, indicating that different subunits respond differentially to AD (25). In simple organisms $d l d$ gene suppression improves stress resistance in insects and increases lifespan in C. elegans $(26,27)$, suggesting that a therapeutic intervention that either suppressed $d l d$ gene expression or DLD enzyme activity could inhibit the symptoms of AD.

5-methoxyindole-2-carboxylic acid (MICA) is a chemical inhibitor of DLD enzyme activity whose effects can be reversed by calcium ionophore-A23187 (CaI), which increases intracellular calcium levels $(28,29)$. In the present study, we diminished the activity of DLD by RNAi or by exposure to MICA in transgenic C. elegans expressing human tau. This allowed us to investigate the possible role of glucose energy metabolism in relation to DLD actvity on tau phosphorylation. We find that increase in whole-body glucose levels that consequently, increases phosphorylation of several serine and threonine residues of tau that are important in NFTs formation after $d l d$ inhibition could be revrsed by $\mathrm{CaI}$. 


\section{Materials and methods}

\section{Nematode strains}

The wild-type strain was N2 (Bristol), whereas transgenic strain VH255 (hdEx82[F25B3::tau352 (WT) + pha-1 (+)] expressing human fetal 352aa CNS tau was used to assess the phosphorylation on tau and maintained as described earlier (30). Although our tau transgenic strain express fetal tau, the sites and magnitude of phosphorylation of fetal tau is similar to PHF-tau in $\mathrm{AD}(31)$.

\section{Culture conditions}

Mixed-stage cultures of $C$. elegans were maintained on nematode growth medium (NGM) seeded with E. coli OP50 at $20^{\circ} \mathrm{C}$. Assays utilised synchronised cultures, which were obtained by harvesting eggs from gravid hermaphrodites by exposing them to a freshly prepared alkaline bleach solution $(0.75 \mathrm{~N} \mathrm{NaOH}+1.5 \mathrm{~N} \mathrm{NaOCl})$. Exposure was for five minutes at room temperature followed by rinsing 3 times with M9 buffer $\left(6 \mathrm{~g} / \mathrm{L} \mathrm{Na}_{2} \mathrm{HPO}_{4} ; 3\right.$ $\mathrm{g} / \mathrm{L} \mathrm{KH} \mathrm{KO}_{4} ; 5 \mathrm{~g} / \mathrm{L} \mathrm{NaCl} ; 0.25 \mathrm{~g} / \mathrm{L} \mathrm{MgSO}_{4} \cdot 7 \mathrm{H}_{2} \mathrm{O}$ ) and centrifugation at $1100 \mathrm{rpm}$ for 1 minute at room temperature. Supernatant was discarded, and egg's pellets were placed freshly made NGM plates at $25^{\circ} \mathrm{C}$ overnight for hatching. Next day, the resulting L1 larvae were shifted to their respective plates. Worms were monitored by visual observation under a microscope, and/ or quantified using the WormScan (32).

\section{dld-1 suppression by RNAi}

The E. coli strain SJJ_LLC1.3 (Source Bioscience) was used to suppress the $d l d-1$ gene in each strain (33). Briefly, feeding bacterial strain was cultured in LB media containing $100 \mu \mathrm{g} /$ $\mathrm{mL}$ ampicillin overnight in shaker at $37^{\circ} \mathrm{C}$ and kept at $4^{\circ} \mathrm{C}$ for further usage. $300 \mu \mathrm{L}$ of feeding bacteria was transferred to NGM plates containing $100 \mu \mathrm{g} / \mathrm{mL}$ ampicillin and $1 \mathrm{mM}$ IPTG. Plates were kept at $25^{\circ} \mathrm{C}$ overnight to grow bacterial cultures. Synchronised worms were shifted to RNAi feeding plates and kept at $20^{\circ} \mathrm{C}$ for 48 hours before using for any assay. 


\section{Chemical inhibition of dld complex in C. elegans using MICA}

As MICA is partially soluble in water, stock solution of MICA was prepared by adding drop wise $5 \mathrm{~N}$ sodium hydroxide till MICA becomes clearly soluble in water.

\section{Acetylcholine induction assay}

Worms with and without $d l d-1$ inhibition were incubated in the presence of $1 \mathrm{mM}$ aldicarb an acetylcholinesterase inhibitor (34) and scored for aldicarb-induced paralysis. Active worms were counted every 30 minutes till last worm became paralyzed.

\section{Thrashing assay}

Thrashing assays were counted in M9 buffer under microscope. Briefly, at least 10 worms were randomly picked from each group and their thrashing rates were counted in a time interval for 10 seconds at $20^{\circ} \mathrm{C}$.

\section{Western Blotting}

To detect phosphorylation on tau protein, synchronised worms were incubated at $20^{\circ} \mathrm{C}$. L4 worms (day 3) were collected and washed three-four times to completely remove the bacterial traces. Half of the washed worms were added in $400 \mu 1$ cold RIPA buffer containing proteinases and phosphatases. While remaining half of the worms were shifted to new plates containing $75 \mu \mathrm{M}$ FUDR to restrict progeny production and collected on $7^{\text {th }}$ day. Worms were subjected to sonication and lysate was collected for further protein analysis. Total protein in the supernatant was measured using Pierce Coomassie (Bradford) protein assay kit (Thermo Scientific) on Nano drop. From each sample, 80-100 $\mu \mathrm{g}$ of total protein was precipitated with acetone and dissolved in Novex® Tricine SDS sample buffer (LC1676, Invitrogen) by heating to $99^{\circ} \mathrm{C}$ for 5 minutes. 50-80ng of whole protein was dissolved in SDS sample buffer and subjected to standard $12.5 \%$ gel electrophoresis. Proteins were transferred to nitrocellulose membrane for $60 \mathrm{~min}$ at $17 \mathrm{~V}$ for semi-dry transfer. Membranes were blocked in $5 \% \mathrm{BSA}$ in $0.1 \%$ TBST containing $0.1 \%$ Tween 20 . A number of antibodies were used to assess total and site specific phosphorylated tau. The antibodies used for tau were, Tau-5 (ab80579, abcam), HT7 (MN1000, Thermo Scientific), phosphor S198 (ab79540, abcam), 
phosphor S235 (NB100-82241, Novus Biologicals), phosphor S262 (79-152, Prosci), phosphor S396 (710298, Novex Lifetecnologies), AT8 (S202, MN1020, Thermo Scientific), and AT180 (T231, MN1040, Thermo Scientific). Anti-actin antibody (2Q1055, abcam) was used as reference control. Anti-mouse IgG alkaline phosphatase antibody produced in goat (A3562, Sigma), and anti-rabbit IgG alkaline phosphatase antibody produced in goat (A3687, Sigma were used as secondary antibody at 1:10000 dilution in 1\% PBS in 0.1\% TBST. 2ndary antibody staining was done for 1 hour at room temperature. After washing the membrane with TBST, the proteins were detected using BCIP/ NBT substrate system (Sigma) or BCIP/ NBT kit (002209) from Lifetechnologies dissolved in 1M Tris (ph 9.0).

\section{Determination of total glucose}

Total glucose concentration in whole worm lysate was determined using Sigma glucose assay kit (GAGO20) under described method. Briefly, transgenic tau VH255 synchronised worms were placed on NGM plates containing different MICA and/ or glucose concentrations at $20^{\circ} \mathrm{C}$. Day 3 worms were collected and washed with M9 buffer to remove any bacterial contents. Worm pellets were dissolved in ice cold RIPA buffer, flash frozen in liquid nitrogen, and placed at $-80^{\circ} \mathrm{C}$ for further analysis. Worms were sonicated and lysate was collected after centrifugation at $10000 \mathrm{rpm}$ at $4{ }^{\circ} \mathrm{C}$. Pellet was discarded. Total protein content was measured using Pierce Coomassie (Bradford) protein assay kit (Thermo Scientific) on Nano drop, and used as normalisation factor. Same lysate was used for total glucose determination in $\mathrm{mg}$ and converted to $\mathrm{mM}(1 \mathrm{mg} / \mathrm{dl}$ glucose $=\sim 5.5 \mathrm{mM})$.

\section{Reversal of MICA-mediated effects}

Transgenic worm VH255 were synchronized and placed at NGM plates containing dld-1 RNAi and/ or $5 \mathrm{mM}$ MICA for 24 hours at $20^{\circ} \mathrm{C}$. L2 staged worms were then shifted to NGM plates containing $35 \mu \mathrm{M}$ calcium ionophore A23187 (CaI) with and without MICA/ dld-1 RNAi. Whole body proteins were extracted from L4 worms and proceed further for analysis.

\section{Statistical analysis}


Mean, standard deviation and $p$ values were calculated using GraphPad (San Diego, CA) prism 6.0.3. Differences between treated and untreated worm strains were analysed for statistical significance by independent student's t-test or one-way Anova. A $p$ value less than 0.05 was considered statistically significant. 


\section{Results}

In this study we used the C. elegans strain that express human fetal tau in neurons. As we are interested to find association between impaired energy metabolism and $\mathrm{AD}$, here in this study we investigated the effects of suppression of core metabolism enzyme DLD on tau-mediated toxicity in $C$. elegans. Tau become phosphorylated in AD that is a complex phenomenon. While hyperphosphotylation is generally associated with toxicity of tau, specific sites may be differentially phosphorylated and may contribute disproportionately to toxicity. Previous studies revealed that Ser-46, Ser-198, Ser-199, Ser-202, Thr-205, thr-212, Thr-231/Ser-235, Ser-262/Ser-356, Ser-396, Ser-404, and Ser-422 are among critical sites in AD and hyperphosphorylation on these residues hinders its binding with microtubules and promote NFTs formation $(9,12,35-37)$. In this study we investigated the effect of $d l d-1$ suppression on tau toxicity and phosphorylation on several critical sites/ epitopes to draw any association between glucose energy metabolism and tau phosphorylation in AD. 

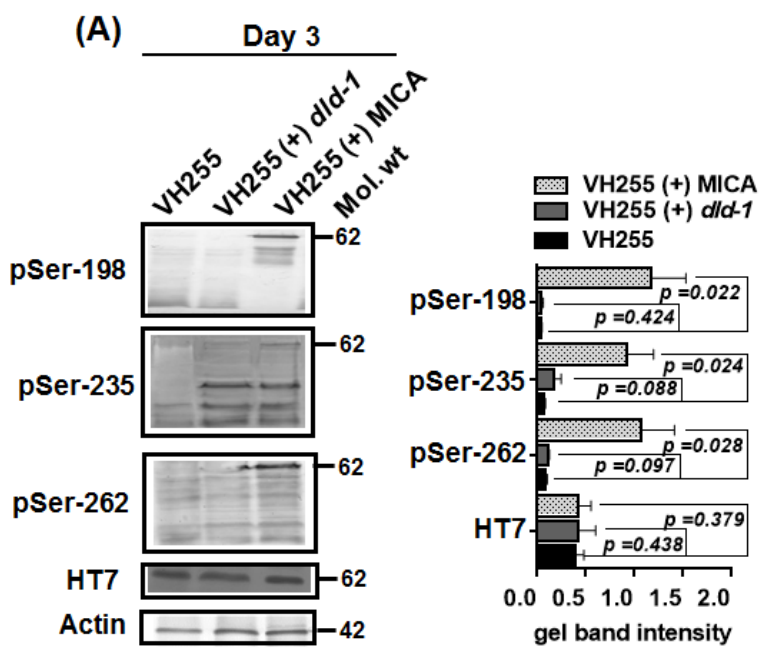

(B)

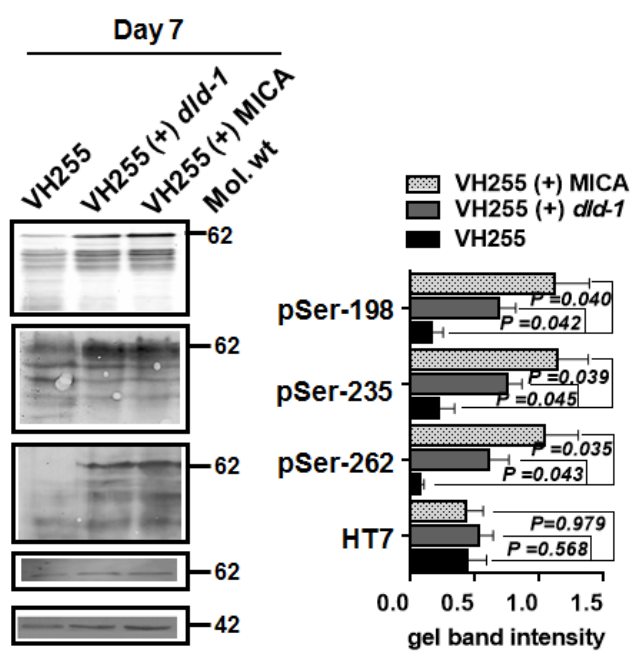

Figure 1: Effect of dld inhibition on tau phosphorylation. Worms were either fed with dld-1 RNAi or with $5 \mathrm{mM}$ MICA after synchronization. Proteins were extracted after three days or seventh day of feeding to check long term effect of $d l d-1$ suppression. Extracted proteins were run on $10 \%$ SDS PAGE and tau was detected by using non-phosphorylated antibody HT-7, and site specific phosphor antibodies Ser-198, Ser-235 and Ser-262. Anti-actin antibody was used as reference control. A) Antibody staining of worm protein extracted after three days. B) Antibody staining of worm protein extracted after seventh day. Quantification of gel bands from each row was done using GelQuantNET. Graphs are shown from two independent trials. Bars $=$ Mean \pm SD.

To check whether inhibition of either the $d l d-1$ gene or DLD protein affects phosphorylation of tau, phosphorylation on specific tau epitopes was analysed using site-specific phospho antibodies on day 3 and day 7 after $d l d-1$ suppression/ inhibition. The HT7 antibody that represent total tau (independent of phosphorylation) showed that inhibition of $d l d-1$ does not changed overall tau levels in worms until day 7. We observed a significant increase in phosphorylation pSer-198 (27.1 fold), pSer-235 (12.85 fold), and pSer-262 (12.3 fold) in MICA (DLD inhibited) treated samples on day 3 that become more prominent on day 7 however, at this time transgenic control also showed phosphorylation. Although on day 3, worms fed with $d l d-1$ RNAi did not showed any significant increase in phosphorylation on any of the sites relative to the untreated transgenic control, by day 7 there was a significant increase in phosphorylation on Ser-198 (4.01 fold), Ser-235 (3.43 fold) and Ser-262 (7.71 fold) epitopes. The most prominent difference in the response to MICA relative to dld-1 RNAi is a difference in the speed of phosphorylation. This is most likely due to direct inhibition of the enzyme being faster than a decrease in gene expression that requires protein turnover to occur before the effect can be observed. 

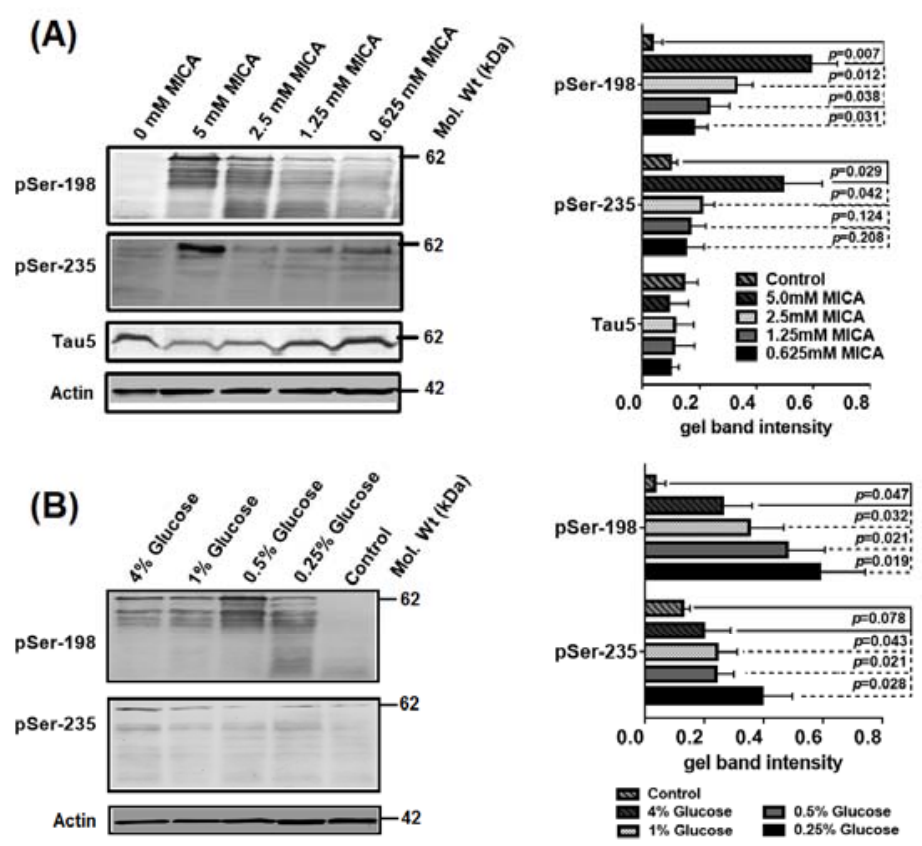

(C)

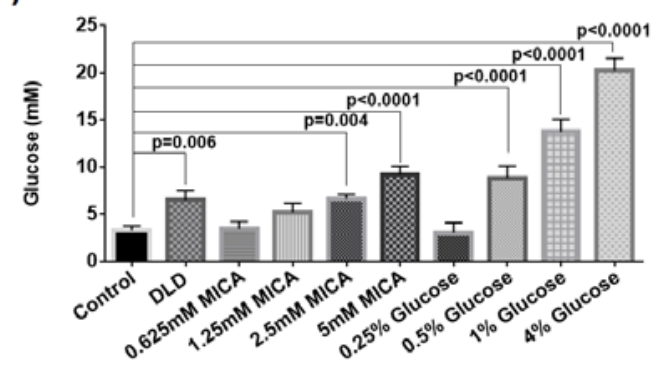

Figure 2: MICA induce tau phosphorylation dose dependantly that can be attributed to induced intracellular glucose levels. (A) Tau expressing worms were synchronised and shifted to $0,5,2.5,1.25$, and $0.625 \mathrm{mM}$ concentrations of MICA. B) To check whether glucose modulate tau phosphorylation, worms were fed with different concentrations of glucose $(0.25,0.5,1$, and $4 \%)$. Proteins were extracted after 3 day incubation and tau phosphorylation was detected. Tau phospho antibodies specific to Ser-198 and Ser-235 were used to detect any changes in tau phosphorylation. Anti- actin antibody was used as refence control. Quantification of gel bands from each row was done using GelQuantNET. Graph are shown from two independent trials. C) Determination of whole body glucose levels in tau transgenic worms. Synchronised worms were shifted to different concentrations of glucose and MICA or fed with dld-1 RNAi. Glucose concentrations were mesured and normalized against extracted protein after 3 days incubation. Glucose concentrations were measured in $\mathrm{mg}$ and then converted to $\mathrm{mM}(1 \mathrm{mg} / \mathrm{dl}$ glucose $=$ $\sim 5.5 \mathrm{mM}$ ). Graph are shown from three independent trials. Bars = Mean \pm SD.

DLD is a part of important enzyme complex PDH that links between glycolysis and Kreb's cycle. Previous studies showed that inhibition of DLD through MICA increased intracellular glucose levels $(28,38)$. Our aim was to evaluate whether this increase in tau phosphorylation in MICA treated worms is linked with increased glucose levels. To assess this, we fed worms with different concentrations of MICA (ranging from $5 \mathrm{mM}-0.625 \mathrm{mM})$ and glucose $(4 \%$ $0.25 \%$ ) separately in NGM. We found that both MICA and glucose increased phosphorylation of tau dose dependantly. Concentrations of MICA 0.625, 1.25, 2.5 and 5mM induce phosphorylation on pSer-198 by 5.1, 6.6, 9.4 and 16.8 fold, and on pSer-235 by 1.5 , 
1.7, 2.1, and 4.9 fold, respectively (Fig 2A). Meanwhile glucose concentrations of $0.25,0.5$, 1 , and $4 \%$ induce phosphorylation on pSer-198 by 7.4, 10.1, 13.6 and 15.6 fold, and on pSer235 by $1.5,1.8,1.8$, and 3.1 fold, respectively (Fig $2 \mathrm{~B}$ ).

Glucose quantification in worms fed with dld-1 RNAi, MICA and glucose showed significant increase in whole body glucose levels (Fig 2C). The glucose concentration in untreated VH255 control worms was $3.35 \pm 0.95 \mathrm{mM}$, while addition of $5 \mathrm{mM}$ MICA in NGM resulted in elevated intracellular glucose concentration $9.12 \pm 0.91 \mathrm{mM}(\mathrm{p}<0.0001)$. Same as MICA, addition of glucose also increased intracellular glucose cencentration where $0.5 \%$ glucose induced intracellular glucose as of $5 \mathrm{mM}$ MICA (8.85 $\pm 1.26, \mathrm{p}=0.729)$. Dld-1 suppression by RNAi also resulted in increase in intracellular glucose that was significantly higher than control $(6.47 \pm 1.05, \mathrm{p}=0.008)$ and almost equal to $1.25 \mathrm{Mm}$ MICA treatment $(5.23 \pm 0.93, \mathrm{p}=0.198)$. 

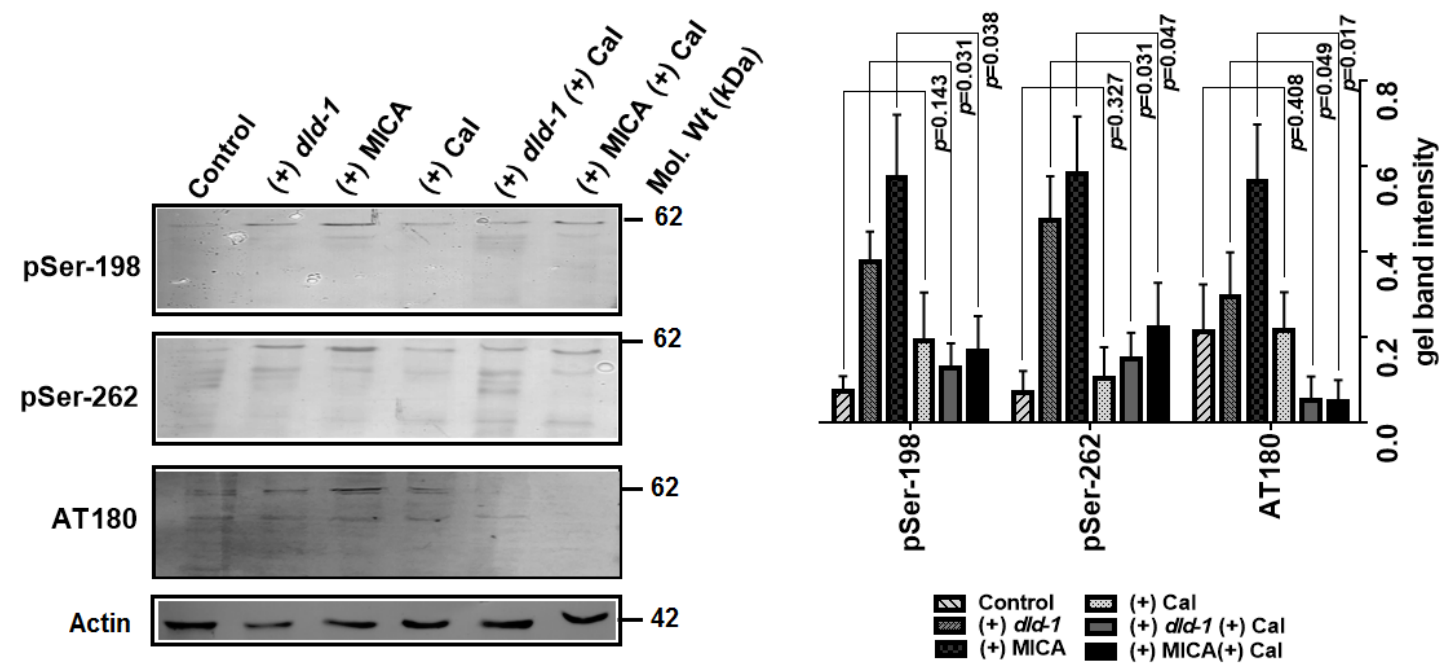

Figure 3: Effect of CaI on tau phosphorylation in presence of $d l d-1$ suppression and DLD inhibition. Tau phosphorylation induced by disruption of DLD may be reduced by CaI. Synchronised tau expressing worms were shifted for 24 hours to $35 \mu \mathrm{M}$ CaI plates with or without dld-1 gene inhibition by RNAi or DLD enzyme inhibition by $5 \mathrm{mM}$ MICA. Whole body protein was extracted from worms after 7 days and evaluated for phosphorylation on respective tau epitopes using antibodies anti Ser-198, Ser-262, AT180.

Quantification of gel bands from each row was done using GelQuantNET. Graph are shown from two independent trials. Bars $=$ Mean \pm SD

Inhibition of DLD was found to induce phosphorylation on all tau epitopes in this study (Fig 1 and 2). As inhibition of DLD activity can be reversed using CaI (29), we assessed whether CaI was able to reverse this induction in phosphorylation in dld-1 suppressed/ DLD inhibited worms. Treatment with CaI reduced tau phosphorylation on pSer-198, pSer-262, and AT180 epitope by $2.9,3.1$, and 5.8 fold in worms fed with dld-1 RNAi, and 3.4, 2.6, and 11.6 fold in MICA fed worms, respectively. CaI as it does not show any difference in tau phosphorylation profile when compared to control. 


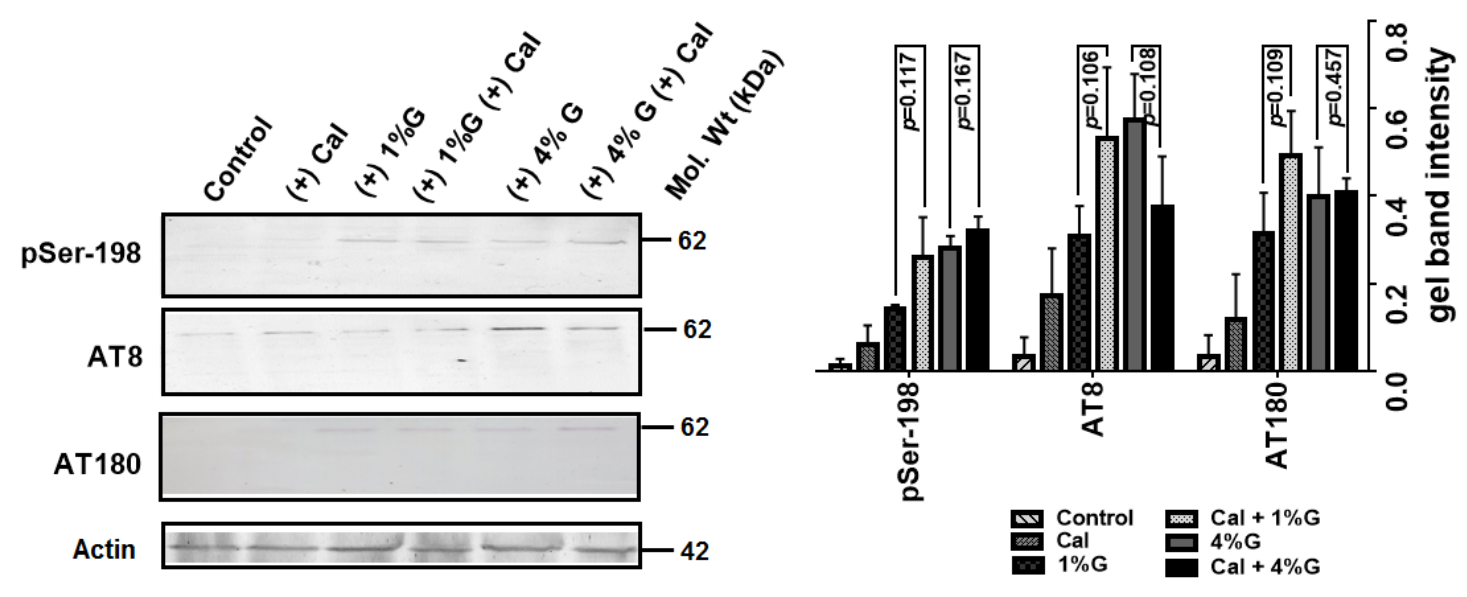

Figure 4: Induction of tau phosphorylation by glucose could not be countered by CaI. Tau expressing worms were fed with $1 \%$ or $4 \%$ glucose in the presence or absence of $35 \mu \mathrm{M}$ CaI. Whole body protein was extracted after 3 days and subjected to immunoblotting using tau phospho epitopes namely Ser-198, AT8 and AT180. Quantification of the intensities of each band relative to actin in each lane was carried out using GelQuantNET software. Graph are shown from two independent trials. Bars $=$ Mean \pm SD

In our study we observed that exposure to a diet supplemented with glucose resulted in induction of tau phosphorylation (Fig 1 and 2) whereas treatment with CaI reduced tau phosphorylation in presence of DLD inhibition (Fig 3). Our aim was to evaluate whether treatment with $\mathrm{CaI}$ reduced the phosphorylation of tau in the presence of high glucose. We found, however, that $\mathrm{CaI}$ was not able to minimize phosphorylation in worms fed with either $1 \%$ or $4 \%$ glucose (Fig 4 ). 


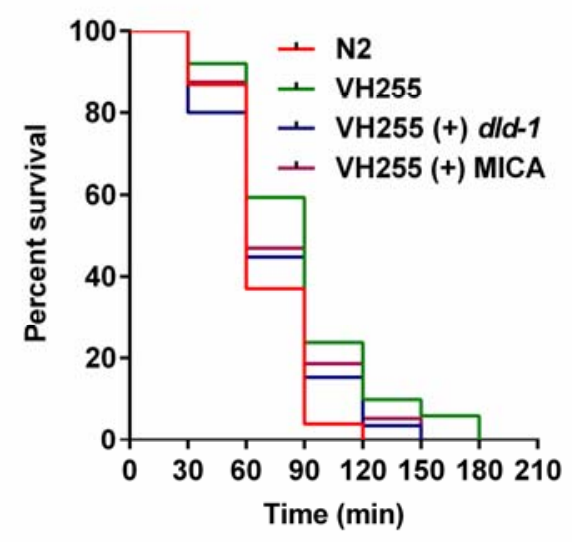

Figure 5: Aldicarb sensitivity assay in wild type and tau expressing worms. Time-dependent paralysis of worms on $1 \mathrm{mM}$ aldicarb fed with and without dld-1 RNAi or $5 \mathrm{mM}$ MICA. Inhibition of either dld-1 gene expression or DLD enzyme activity by MICA modulates acetylcholine neurotransmission. Paralysis curves were compared using Log-rank method. Results were derived from three independent experiments.

In this study we revealed that dld- 1 suppression and DLD inhibition increased tau phosphorylation that could result in induced tau pathology. Transgenic tau expression in worms was found negatively affects the synaptic transmission of cholinergic neurons and induced resistance against acetylcholine esterase (AChE) inhibitor aldicarb when compared to wild type (39). To check whether $d l d-1$ suppression or inhibition could affect acetylcholine neurotransmission, we treated the worms with aldicarb. In our study, when treated with aldicarb, wild type N2 worms become paralysed within 120 minutes when compared to tau expressing VH255 strain that took 180 minutes to become paralysed ( $<<0.0001)$. We found earlier paralysis in tau expressing worms compared to transgenic control in the presence of aldicarb when fed with either dld-1 RNAi (150 min, $\mathrm{p}=0.0131)$ or MICA (150 min, $\mathrm{p}=0.0272$ ). The reduction in paralysis time by $d l d-1$ RNAi was not significantly different from suppression of enzyme activity by MICA ( $\mathrm{p}=0.557)$. Although suppression of $d l d-1$ was able to reduce aldicarb resistance in transgenic worms, it was not up to the wild type ( $d l d-1$ RNAi, 120 vs $150 \mathrm{~min}, \mathrm{p}=0.0431$; MICA, 120 vs $150 \mathrm{~min}, \mathrm{p}=0.0038)$. Thus, restoration of normal acetylcholine signalling is the only AD-like symptom that can be alleviated by $d l d-1$ suppression in the C. elegans tau model of AD. 


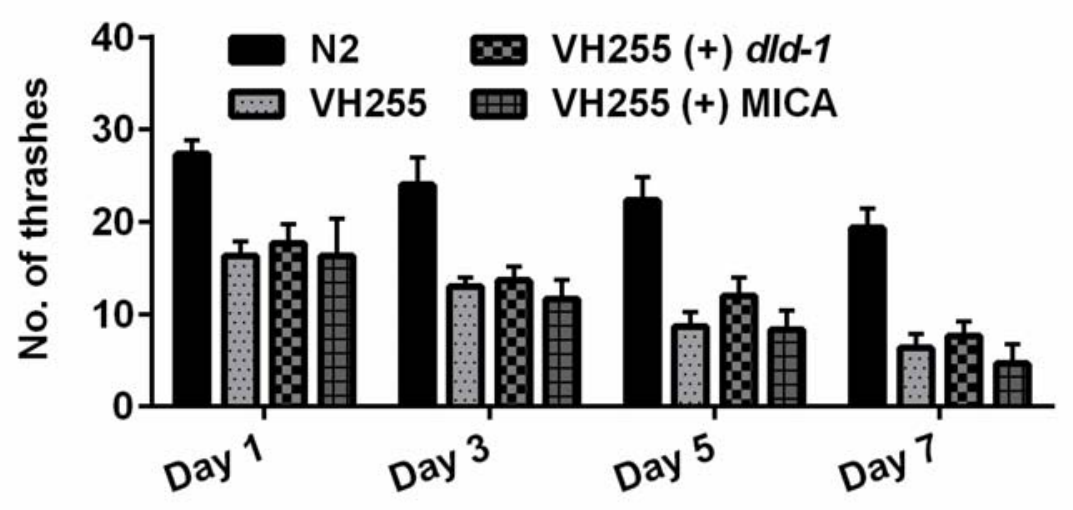

Figure 6: Inhibition of either $d l d-1$ gene expression or DLD enzyme activity does not restore thrashing rates of tau expressing worms. Quantification of thrashing rates in tau expressing worms (VH255) with and without dld-1 gene or DLD enzyme suppression. Thrashing rates were quantified every second day from the start of the experiment until day 7 and compared with wild type (N2) worms that did not express tau.

Improvements in acetylcholine neurotransmission could affect the worm locomotion. When submerged in liquid, C. elegans will move vigorously, but will be unable to gain traction as they would on a solid surface. This is referred to as thrashing. Expression of human tau in $C$. elegans resulted in reduced thrashing rates (30). Suppression of either the $d l d-1$ gene by RNAi or the DLD enzyme by exposure to MICA failed to restore normal movement. We monitored the thrashing rates till day seven and observed no improvement. Thrashing rates decreased gradually over the course of the experiment irrespective of treatment or genotype. 


\section{Discussion}

In this study we examined the effect of suppression of $d l d-1$ gene expression or chemical inhibition of the DLD enzyme activity on phosphorylation of the human tau protein in a $C$. elegans model of Alzheimer's disease. As inhibition of either the dld gene or enzyme activity significantly disrupts glucose catabolism, we also explored the relationship between glucose levels and tau phosphorylation. We then used movement and neural function assays to monitor the relationship between tau phosphorylation and pathology. DLD is a subunit of $\mathrm{PDH}$, an enzyme complex that regulates glucose catabolism at the interface between glycolysis and the TCA cycle (40). MICA is a chemical inhibitor of DLD that we used in this study. In $H$. volcanii and in mouse models, MICA does not exclusively inhibit PDH as described previously $(28,41)$, but also dehydrogenases of other substrates including succinate, lactate and alpha-ketoglutarate. Therefore, to complement our studies with MICA, we also carried out specific epigenetic suppression of the $d l d-1$ gene.

Inhibition of DLD and subsequently other mitochondrial dehydrogenase may lead to rise in glucose concentrations by reducing the carbon of pyruvate into the citric acid cycle (42) and might causing hyperglycaemia like stage in worms. Hyperglycaemia increases phosphorylation of tau in mouse models of diabetes (43-45). As DLD is a subunit of PDH, an enzyme that regulates glucose catabolism, we reasoned that inhibition of either DLD enzyme activity or expression of the $d l d-1$ gene would decrease the glycolytic catabolism of glucose (42) causing a rise in glucose concentration in C. elegans. In our study, inhibition of DLD by MICA resulted in an increase in phosphorylation at multiple sites of the tau protein. The response to dld-1 gene suppression was slower, but after 7 days, a similar increase in phosphorylation at multiple sites of the tau protein occurred (Fig 1). The response to MICA was dose-dependent and as exposure to MICA and dld-l gene suppression both caused whole-body levels of glucose to increase, the effect of glucose on phosphorylation of tau was also determined (Fig 2). Like the situation in response to MICA exposure, the response to glucose was also seemed dose dependently.

$\mathrm{CaI}$ is reported to act as a MICA antagonist $(46,47) . \mathrm{CaI}$ is known to decrease the phosphorylation of the PDH enzyme complex and regulate glucose transport to cells (48-52). In our study, $\mathrm{CaI}$ alone did not affect the overall pattern of tau phosphorylation but it did prevent induction of phosphorylation by either $d l d-1$ RNAi or exposure to MICA (Fig 3). Interestingly, CaI failed to reduce tau phosphorylation in the presence of high glucose (Fig 4). 
The failure to prevent the phosphorylation due to elevated glucose in the diet was surprising given the ability of $\mathrm{CaI}$ to prevent phosphorylation due to inhibition of the $d l d-1$ gene or DLD enzyme. This result indicates either that the phosphorylation occurs by different mechanisms or that the effect of CaI is mediated downstream of DLD, but upstream of glucose in a response pathway.

Expression of tau in C. elegans results in impaired cholinergic neurotransmission (39, 53) and progressive aged-dependent uncoordinated movement. Resistance against acetylcholinesterase inhibitor "aldicarb", may be due to either a pre- or post-synaptic defect (54). Treatment with aldicarb causes more severe muscle contraction and early paralysis in wild type $C$. elegans compared to tau transgenic strains. Although the mechanism is unknown, both pre and post-synaptic defects observed in tau transgenic control were partially reversed by $d l d-1$ inhibition either using RNAi or MICA (Fig 5). Improvement in acetylcholine neurotransmission could improve worm's uncoordinated movement. We found that $d l d-1$ inhibition did not reduce uncoordinated movement and that the thrashing rates decreased gradually with age (Fig 6). These results are consistent with a previously published report that attributed impaired movement to microtubules being "overstabilzed" by the expression of transgenic tau (30). As both $d l d-1$ gene suppression and treatment with MICA increase phosphorylation of tau without affecting the amount of tau protein, the ameliorative effect seems to be independent of tau or the general phosphorylation state of the protein. These results are in agreement with previous findings in which early staged-synaptic regulation and uncoordinated movement was independent of tau phosphorylation $(30,55)$.

In conclusions, we found that impairment of the enzyme DLD, which is involved in glucose energy metabolism, significantly induces phosphorylation of the tau protein. The outcome was the same whether impairment was by post-transcriptional silencing of the $d l d-1$ gene or by inhibition of the DLD enzyme by MICA. The phosphorylation induced by either method was reversible by the CaI. Both MICA and $d l d-1$ gene suppression resulted in elevated levels of whole-body glucose. While elevated glucose also resulted in increased phosphorylation of the tau protein, this effect could not be reversed by exposure to the CaI. Our results suggest that glucose-mediated phosphorylation occurs by a different mechanism or that it is mediated downstream of $\mathrm{Ca}^{2+}$ signalling. 


\section{References}

1. Friedland-Leuner K, Stockburger C, Denzer I, Eckert GP, \& Muller WE (2014) Mitochondrial dysfunction: cause and consequence of Alzheimer's disease. Progress in molecular biology and translational science 127:183-210.

2. Chaturvedi RK \& Flint Beal M (2013) Mitochondrial diseases of the brain. Free radical biology \& medicine 63:1-29.

3. Zhao W, et al. (2015) Impaired mitochondrial energy metabolism as a novel risk factor for selective onset and progression of dementia in oldest-old subjects. Neuropsychiatric disease and treatment 11:565-574.

4. Kalpouzos G, et al. (2009) Voxel-based mapping of brain gray matter volume and glucose metabolism profiles in normal aging. Neurobiology of aging 30(1):112-124.

5. Bubber P, Haroutunian V, Fisch G, Blass JP, \& Gibson GE (2005) Mitochondrial abnormalities in Alzheimer brain: mechanistic implications. Annals of neurology 57(5):695-703.

6. Gibson GE, et al. (2012) Deficits in the mitochondrial enzyme alpha-ketoglutarate dehydrogenase lead to Alzheimer's disease-like calcium dysregulation. Neurobiology of aging 33(6):1121 e1113-1124.

7. Hardas SS, et al. (2013) Oxidative modification of lipoic acid by HNE in Alzheimer disease brain. Redox biology 1:80-85.

8. Gibson GE, Park LC, Sheu KF, Blass JP, \& Calingasan NY (2000) The alpha-ketoglutarate dehydrogenase complex in neurodegeneration. Neurochemistry international 36(2):97-112.

9. Iqbal K, Liu F, Gong CX, Alonso Adel C, \& Grundke-Iqbal I (2009) Mechanisms of tau-induced neurodegeneration. Acta neuropathologica 118(1):53-69.

10. Iqbal K, et al. (2010) Alzheimer's disease neurofibrillary degeneration: pivotal and multifactorial. Biochemical Society transactions 38(4):962-966.

11. Iqbal K, Liu F, Gong CX, \& Grundke-lqbal I (2010) Tau in Alzheimer disease and related tauopathies. Current Alzheimer research 7(8):656-664.

12. Wang JZ, Xia YY, Grundke-lqbal I, \& Iqbal K (2013) Abnormal hyperphosphorylation of tau: sites, regulation, and molecular mechanism of neurofibrillary degeneration. Journal of Alzheimer's disease : JAD 33 Suppl 1:S123-139.

13. Idan-Feldman A, Ostritsky R, \& Gozes I (2012) Tau and caspase 3 as targets for neurop rotection. International journal of Alzheimer's disease 2012:493670.

14. Iqbal K, Gong CX, \& Liu F (2013) Hyperphosphorylation-induced tau oligomers. Frontiers in neurology 4:112.

15. Liu F, Iqbal K, Grundke-Iqbal I, Hart GW, \& Gong CX (2004) O-GIcNAcylation regulates phosphorylation of tau: a mechanism involved in Alzheimer's disease. Proceedings of the National Academy of Sciences of the United States of America 101(29):10804-10809.

16. Robertson LA, Moya KL, \& Breen KC (2004) The potential role of tau protein O-glycosylation in Alzheimer's disease. Journal of Alzheimer's disease : JAD 6(5):489-495.

17. Liu Y, Liu F, Grundke-Iqbal I, Iqbal K, \& Gong CX (2009) Brain glucose transporters, OGlcNAcylation and phosphorylation of tau in diabetes and Alzheimer's disease. Journal of neurochemistry 111(1):242-249.

18. Deng Y, et al. (2009) Dysregulation of insulin signaling, glucose transporters, OGlcNAcylation, and phosphorylation of tau and neurofilaments in the brain: Implication for Alzheimer's disease. The American journal of pathology 175(5):2089-2098.

19. Sun J, Feng X, Liang D, Duan Y, \& Lei H (2012) Down-regulation of energy metabolism in Alzheimer's disease is a protective response of neurons to the microenvironment. Journal of Alzheimer's disease : JAD 28(2):389-402.

20. Lee SJ, Murphy CT, \& Kenyon C (2009) Glucose shortens the life span of C. elegans by downregulating DAF-16/FOXO activity and aquaporin gene expression. Cell metabolism 10(5):379-391. 
21. Schulz TJ, et al. (2007) Glucose restriction extends Caenorhabditis elegans life span by inducing mitochondrial respiration and increasing oxidative stress. Cell metabolism 6(4):280293.

22. Peters A (2011) The selfish brain: Competition for energy resources. American journal of human biology : the official journal of the Human Biology Council 23(1):29-34.

23. Schlipalius DI, et al. (2012) A core metabolic enzyme mediates resistance to phosphine gas. Science 338(6108):807-810.

24. Brown AM, et al. (2007) Testing for linkage and association across the dihydrolipoyl dehydrogenase gene region with Alzheimer's disease in three sample populations. Neurochemical research 32(4-5):857-869.

25. Gibson GE, et al. (1998) Alpha-ketoglutarate dehydrogenase in Alzheimer brains bearing the APP670/671 mutation. Annals of neurology 44(4):676-681.

26. Cheng $Q$, et al. (2003) Caenorhabditis elegans mutants resistant to phosphine toxicity show increased longevity and cross-resistance to the synergistic action of oxygen. Toxicological sciences : an official journal of the Society of Toxicology 73(1):60-65.

27. Butler JA, Mishur RJ, Bhaskaran S, \& Rea SL (2013) A metabolic signature for long life in the Caenorhabditis elegans Mit mutants. Aging cell 12(1):130-138.

28. Bauman N \& Hill CJ (1968) Inhibition of gluconeogenesis and alpha-keto oxidation by 5methoxyindole-2-carboxylic acid. Biochemistry 7(4):1322-1327.

29. Panneerdoss S, Siva AB, Kameshwari DB, Rangaraj N, \& Shivaji S (2012) Association of lactate, intracellular $\mathrm{pH}$, and intracellular calcium during capacitation and acrosome reaction: contribution of hamster sperm dihydrolipoamide dehydrogenase, the E3 subunit of pyruvate dehydrogenase complex. Journal of andrology 33(4):699-710.

30. Brandt R, Gergou A, Wacker I, Fath T, \& Hutter H (2009) A Caenorhabditis elegans model of tau hyperphosphorylation: induction of developmental defects by transgenic overexpression of Alzheimer's disease-like modified tau. Neurobiology of aging 30(1):22-33.

31. Agnes K \& Shu-Hui C, Yen. (1993) The extent of phosphorylation of fetal tau is comparable to that of PHF-tau from Alzheimer paired helical filaments. Brain Research 26(629):40-46.

32. Mathew MD, Mathew ND, \& Ebert PR (2012) WormScan: a technique for high-throughput phenotypic analysis of Caenorhabditis elegans. PloS one 7(3):e33483.

33. Kamath RS \& Ahringer J (2003) Genome-wide RNAi screening in Caenorhabditis elegans. Methods 30(4):313-321.

34. Mahoney TR, Luo S, \& Nonet ML (2006) Analysis of synaptic transmission in Caenorhabditis elegans using an aldicarb-sensitivity assay. Nature protocols 1(4):1772-1777.

35. Gong CX \& Iqbal K (2008) Hyperphosphorylation of microtubule-associated protein tau: a promising therapeutic target for Alzheimer disease. Current medicinal chemistry 15(23):2321-2328.

36. Iqbal K \& Grundke-lqbal I (2006) Discoveries of tau, abnormally hyperphosphorylated tau and others of neurofibrillary degeneration: a personal historical perspective. Journal of Alzheimer's disease : JAD 9(3 Suppl):219-242.

37. Wang JZ, Grundke-Iqbal I, \& Iqbal K (2007) Kinases and phosphatases and tau sites involved in Alzheimer neurofibrillary degeneration. The European journal of neuroscience 25(1):59-68.

38. Bauman N \& Pease BS (1969) Effects of 5-methoxyindole-2-carboxylic acid on carbohydrate metabolism. Biochemical pharmacology 18(5):1093-1101.

39. Kraemer BC, et al. (2003) Neurodegeneration and defective neurotransmission in a Caenorhabditis elegans model of tauopathy. Proceedings of the National Academy of Sciences of the United States of America 100(17):9980-9985.

40. Babady NE, Pang YP, Elpeleg O, \& Isaya G (2007) Cryptic proteolytic activity of dihydrolipoamide dehydrogenase. Proc Natl Acad Sci U S A 104(15):6158-6163. 
41. Meury J (1993) 5-Methoxyindole-2-carboxylic acid is a potent inhibitor of respiration and potassium ion transport in the archaebacterium Haloferax volcanii. FEMS Microbiology Letters 108(3):271-274.

42. Jeoung NH \& Harris RA (2010) Role of pyruvate dehydrogenase kinase 4 in regulation of blood glucose levels. Korean diabetes journal 34(5):274-283.

43. Clodfelder-Miller BJ, Zmijewska AA, Johnson GV, \& Jope RS (2006) Tau is hyperphosphorylated at multiple sites in mouse brain in vivo after streptozotocin-induced insulin deficiency. Diabetes 55(12):3320-3325.

44. Freude $\mathrm{S}$, et al. (2005) Peripheral hyperinsulinemia promotes tau phosphorylation in vivo. Diabetes 54(12):3343-3348.

45. Kim B, Backus C, Oh S, Hayes JM, \& Feldman EL (2009) Increased tau phosphorylation and cleavage in mouse models of type 1 and type 2 diabetes. Endocrinology 150(12):5294-5301.

46. Tretter L \& Adam-Vizi V (2005) Alpha-ketoglutarate dehydrogenase: a target and generator of oxidative stress. Philosophical transactions of the Royal Society of London. Series $B$, Biological sciences 360(1464):2335-2345.

47. Denton RM (2009) Regulation of mitochondrial dehydrogenases by calcium ions. Biochimica et biophysica acta 1787(11):1309-1316.

48. Sweet IR \& Gilbert M (2006) Contribution of calcium influx in mediating glucose-stimulated oxygen consumption in pancreatic islets. Diabetes 55(12):3509-3519.

49. Sudo Y \& Mariash CN (1996) Lowering glucose depletes a thapsigargin-sensitive calcium pool and inhibits transcription of the S14 gene. Endocrinology 137(11):4677-4684.

50. Resendez E, Jr., Ting J, Kim KS, Wooden SK, \& Lee AS (1986) Calcium ionophore A23187 as a regulator of gene expression in mammalian cells. The Journal of cell biology 103/6 Pt 1):2145-2152.

51. Mitani Y, Behrooz A, Dubyak GR, \& Ismail-Beigi F (1995) Stimulation of GLUT-1 glucose transporter expression in response to exposure to calcium ionophore A-23187. The American journal of physiology 269(5 Pt 1):C1228-1234.

52. Drummond IA, Lee AS, Resendez E, Jr., \& Steinhardt RA (1987) Depletion of intracellular calcium stores by calcium ionophore A23187 induces the genes for glucose-regulated proteins in hamster fibroblasts. The Journal of biological chemistry 262(26):12801-12805.

53. Fatouros C, et al. (2012) Inhibition of tau aggregation in a novel Caenorhabditis elegans model of tauopathy mitigates proteotoxicity. Human molecular genetics 21(16):3587-3603.

54. Nonet ML, Grundahl K, Meyer BJ, \& Rand JB (1993) Synaptic function is impaired but not eliminated in C. elegans mutants lacking synaptotagmin. Cell 73(7):1291-1305.

55. Asuni AA, Perry VH, \& O'Connor V (2010) Change in tau phosphorylation associated with neurodegeneration in the ME7 model of prion disease. Biochemical Society transactions 38(2):545-551. 